\title{
Mesostructure of Mesoporous Silica/Anodic Alumina Hierarchical Membranes Tuned with Ethanol
}

\author{
Silo Meoto, ${ }^{\circledR}$ Niall Kent, Michael M. Nigra, ${ }^{\dagger}$ and Marc-Olivier Coppens* \\ Department of Chemical Engineering, University College London, Torrington Place, London, United Kingdom WC1E 7JE \\ Supporting Information
}

ABSTRACT: Hierarchically structured membranes composed of mesoporous silica embedded inside the channels of anodic alumina (MS-AAM) were synthesized using the aspiration method. Ethanol is shown to have a significant effect on the type and organization of the mesoporous silica phase. Detailed textural analysis revealed that the pore size distribution of the mesoporous silica narrows and the degree of ordering increases with decreasing ethanol concentration used in the synthesis mixture. The silica mesopores were synthesized with pores as small as $6 \mathrm{~nm}$ in diameter, with the channel direction oriented in lamellar, circular, and columnar directions depending on the ethanol content. This study reveals ethanol concentration as a key factor behind the synthesis of an ordered mesoporous silica-anodic alumina membrane that can increase its functionality for membranebased applications.

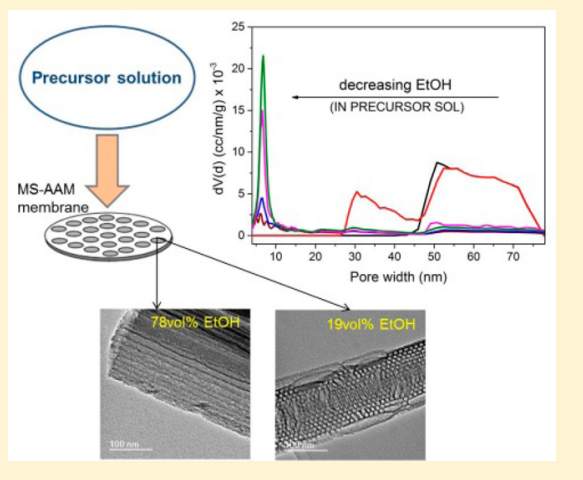

\section{INTRODUCTION}

Since their discovery in the early $1990 \mathrm{~s},{ }^{1-4}$ ordered mesoporous silica materials have gained tremendous interest. This interest stems from their desirable properties, such as welldefined and uniform pore sizes, high surface areas, and high pore volumes. As a result, they are very attractive to a wide range of applications, including catalysis, ${ }^{5-7}$ adsorption, ${ }^{8,9}$ nanotechnology, ${ }^{10,11}$ sensing, ${ }^{12,13}$ and separation. ${ }^{14,15}$ They are typically synthesized via a technique that combines solgel chemistry and the self-assembly of surfactant molecules as templates $^{16}$ in the presence of an acid $^{17}$ or base catalyst. ${ }^{18}$ Significant effort has been expended to understand the formation mechanism and to control the morphology and pore size of mesoporous silica materials by adjusting the synthesis conditions and components. Different surfactants and synthesis conditions lead to different mesostructures, such as lamellar, hexagonal, and cubic phases. ${ }^{4,19-21}$ Block copolymer micelles are a popular choice as a template for the synthesis of mesoporous silica. Unlike low-molecular-weight surfactants, block copolymers yield more stable materials, with thicker silica walls and larger pore diameters. ${ }^{22}$ The most frequently used block copolymer is Pluronic P123 $\left(\mathrm{PEO}_{20}-\mathrm{PPO}_{70}-\mathrm{PEO}_{20}\right)$, which is used in the synthesis of SBA-15 to yield a twodimensionally, hexagonally ordered $(p 6 \mathrm{~mm})$ pore structure with tunable mesopore diameter. ${ }^{3}$

In the presence of alcohols, the behavior of micelles formed by the surfactants is affected. ${ }^{23}$ Alcohols of medium chain length ( $n$-propanol to $n$-hexanol) were reported to be partitioned between micelles and the surrounding aqueous phase, ${ }^{24}$ which leads to mixed alcohol/surfactant micelles. In 1981, Zana et al. $^{23}$ proposed three relaxation processes that characterize an alcohol/surfactant micelle system: a slow process, which corresponds to the mixed micelle formation- dissolution equilibrium and two fast processes, associated with the exchange of the alcohol and surfactant between micelles and the surrounding solution. The authors showed that the addition of linear alcohols (butanol to $n$-hexanol) to micellar solutions decreases the critical micelle concentration (CMC) and molecular weight at the CMC and increases the degree of micelle ionization at the CMC. Examples of materials resulting from the addition of linear alcohols can be seen in the butanolP123 system (cubic Ia3d mesostructure) ${ }^{25}$ and butanol-F127 system (cubic $\operatorname{Im} 3 m, F m 3 m$, and a $2 \mathrm{D}$ hexagonal-like mesophase). ${ }^{26}$ In contrast, short-chain alcohols (methanol and ethanol) have been described as having a water-structurebreaking effect on copolymers, whose addition results in increased copolymer solubility and, therefore, the CMC. ${ }^{23,27-29}$ Denkova et al. ${ }^{30}$ confirmed that the addition of ethanol to an aqueous micellar solution initiates micelle growth and the transition from spheres to rods. The growth process of the micelles was reported to have two distinct regions, which are dependent on the experimental conditions. This process is characterized by an initiation period, followed by a period of actual growth to equilibrium. The reactant solution for the synthesis of SBA-15 usually does not contain additional alcohol, although Sun et al. used alcohol in a thermal treatment to produce a secondary SBA-15 material with long-range ordering, larger mesopores, and thicker pore walls. ${ }^{31}$ Under synthesis conditions without alcohol, ethanol is formed by the hydrolysis of the silicate source, tetraethylorthosilicate (TEOS). The amount of ethanol released is significant enough to drive a phase transformation in the mesoporous silica formed, by

Received: February 9, 2017

Revised: April 20, 2017

Published: April 24, 2017 
altering the surfactant packing within the micellar surfactant templates. $^{32}$ It has been shown that increasing ethanol concentration results in a silica mesophase transition sequence in the order of hexagonal $\rightarrow$ cubic $\rightarrow$ lamellar $\rightarrow$ radial hexagonal close packing for cationic ${ }^{33}$ and anionic ${ }^{34}$ surfactant systems. The amount of alcohol present affects the hydrolysis rate so that, at lower concentrations, the alcohol behaves as a cosurfactant. At higher concentrations, however, the alcohol acts as a cosolvent. As a cosurfactant, the alcohol molecules penetrate the surfactant micelles so that the true volume occupied by the surfactant increases, which results in an increase in the surfactant packing parameter value $(g)$ and a resulting decrease in the surface curvature (hexagonal $\rightarrow$ cubic $\rightarrow$ lamellar). ${ }^{33,35}$

Ethanol is known to be a good solvent for the PEO and PPO blocks in Pluronic P123 block copolymer. As a result, ethanol has a significant influence on the self-assembly behavior of P123 in aqueous solution. Soni et al. ${ }^{29}$ hypothesized that ethanol diffuses inside the PPO core, which leads to a steady decrease in the micellar radius, core size, and aggregation number of the block copolymer micelles as the ethanol concentration increases from 0 to $20 \mathrm{wt} \%$. SAXS data analysis also showed that the mean intermicellar distance decreases with ethanol addition. For 0 and 5 wt \% ethanol concentrations in 30 wt \% P123 copolymer, a mixture of cubic $(\mathrm{Fm} 3 \mathrm{~m})$ and hexagonal close-packed sphere (HCPS) crystalline phases were resolved by SAXS Bragg reflection peaks. For 10 wt \% ethanol in the same copolymer concentration, only the 3D hexagonal HCPS phase was observed. No studies were carried out beyond $25 \mathrm{wt}$ $\%$ ethanol concentration. However, a study of the binary system of P123 and water revealed a transition of crystalline phases from a mixture of cubic and HCPS, to 2D hexagonal to lamellar, when the copolymer concentration increased from 30 to 45 to 70 wt $\%$, respectively. Liu et al. ${ }^{33}$ confirmed that different phase transitions can be induced by the presence of alcohol. Studying a CTAB surfactant-based system, the authors explained the phase transition by a change in the surfactant packing parameter. At low alcohol concentrations, the packing parameter increases as a consequence of an increase in the true surfactant volume when the alcohol diffuses into the surfactant micelles. The increased parameter value leads to a high- to lowcurvature phase transition. This is the cosurfactant effect. Contributing information to the solution properties of PEOPPO-PEO micelles ${ }^{36,37}$ has received a lot of attention and shows that increasing the number of ethylene oxide groups in a surfactant molecule leads to a smaller aggregation number, that is, the number of surfactant molecules present in a micelle once the CMC value has been reached.

For separation applications, mesoporous silica has been grown inside the channels of a hard template so that the pore channels are aligned along the substrate channels; growing mesoporous silica directly as a film will lead to pores parallel to the film's external surface. ${ }^{38,39}$ The synthesis of mesoporous silica inside the columnar channels of an anodic alumina membrane (AAM) has been extensively studied. ${ }^{40-43}$ An anodic alumina membrane is an ideal hard template for the growth of molecular filters consisting of ordered mesoporous silica because of its chemically and mechanically stable nature and straight tunable pore channels that can also be used for separation applications. ${ }^{44,45}$ However, control over the formation of the silica structures in this confined environment is still difficult. Here, the aspiration method is used as the fabrication method for the synthesis of mesoporous silica with controlled morphology and a tunable pore size distribution. This method has been previously reported ${ }^{46}$ to be superior to other evaporation-induced self-assembly (EISA) techniques. A key factor that can be manipulated in this technique to create ordered mesoporous structures inside the AAM channels is the residence time for the precursor sol. It is the purpose of this article to investigate the influence of ethanol on the growth of mesoporous silica structures inside AAM channels. As discussed, ethanol is a common organic solvent used in the synthesis of mesoporous silica materials. However, unlike the silica sol for granular mesoporous silica, the silica sol for mesoporous silica membranes requires an additional amount of ethanol, an important difference. In studies of Pluronic P123 micelles, it has been shown that the maximum length of rodshaped micelles is reached at $8-10 \mathrm{vol} \%$ ethanol, ${ }^{47}$ which is equivalent to the amount of ethanol released in the hydrolysis of TEOS during the synthesis of SBA-15, without ethanol added. Therefore, an understanding of why additional ethanol is needed for membrane synthesis and how it affects the synthesis of mesoporous silica is critical and necessary.

In this study, we examine the effect of a wide range of ethanol concentrations on the final mesoporous structures, softtemplated by Pluronic P123 and formed within the channels of anodic alumina. Composite mesoporous silica-anodic alumina membranes were synthesized by varying the ethanol content. The growth process and final synthesized structures as a function of ethanol present in the sol were investigated. This offers crucial new insights to control the synthesis of tunable mesoporous silica membranes, which has remained a great challenge up to now. ${ }^{46}$

\section{EXPERIMENTAL SECTION}

2.1. Materials. Tetraethylorthosilicate (TEOS, reagent grade 98\%) was used as the silica source, and ethanol (absolute), hydrochloric acid $(\mathrm{HCl}, \mathrm{ACS}$ reagent $37 \%)$, and triblock copolymer poly(ethylene oxide)-b-poly(propylene oxide)- $b$-poly(ethylene oxide) $\mathrm{EO}_{20} \mathrm{PO}_{70} \mathrm{EO}_{20}$ (Pluronic P123) were obtained from Sigma-Aldrich Company Ltd. (Dorset, U.K.). Whatman anodic alumina membranes (AAM) with a pore size of $200 \mathrm{~nm}$, a diameter of $47 \mathrm{~mm}$, and a thickness of $60 \mu \mathrm{m}$ were obtained from Fisher Scientific Ltd. (Leicestershire, U.K.). Proteins myoglobin ( $\mathrm{Mb}$, molecular weight $17000 \mathrm{~g} / \mathrm{mol}$ and size $2.9 \times 3.6 \times 6.4 \mathrm{~nm}^{3}$ ) and bovine serum albumin (BSA, molecular weight $66500 \mathrm{~g} / \mathrm{mol}$ and size $5.5 \times 5.6 \times 12 \mathrm{~nm}^{3}$ ) were also obtained from Sigma-Aldrich Company Ltd. (Dorset, U.K.). All materials were used as received without any further purification.

2.2. Synthesis of a Nanocomposite Membrane. A standard precursor solution was prepared as described previously, ${ }^{48}$ with modifications as follows: Pluronic P123 ( $1 \mathrm{~g})$ was dissolved in ethanol with deionized water $(2 \mathrm{~g})$ and hydrochloric acid $(12 \mathrm{M} \mathrm{HCl}, 0.1 \mathrm{~g})$. The solution was stirred at room temperature under reflux for $1 \mathrm{~h}$ until it was clear. Then tetraethylorthosilicate (TEOS, $2.13 \mathrm{~g}$ ) was added to the mixture and stirred for $7 \mathrm{~h}$ at $60{ }^{\circ} \mathrm{C}$ under reflux. The molar composition of the precursor solution, TEOS $/ \mathrm{P} 123 / \mathrm{EtOH} / \mathrm{HCl} / \mathrm{H}_{2} \mathrm{O}$ is $1: 0.017: x: 0.1: 11.2$. Table 1 shows the molar composition of the precursor solution used to create the different membrane samples.

An anodic alumina membrane (AAM) was set in a filtration apparatus, and $5 \mathrm{~mL}$ of the precursor solution was dropped onto the membrane. The solution was forced into the channels under moderate aspiration of $20 \mathrm{kPa}$ (vacuum pressure). The membrane was dried under aspiration for $5 \mathrm{~min}$ at room temperature. To remove the surfactant template, the sample was calcined at $550{ }^{\circ} \mathrm{C}$ for $6 \mathrm{~h}$ after increasing the temperature at a heating rate of $0.5^{\circ} \mathrm{C} / \mathrm{min}$ from room temperature to $550{ }^{\circ} \mathrm{C}$. Hereafter, the resulting mesoporous membranes prepared will be designated as $X \_M$, where $X$ is the volume percent of ethanol added to the precursor solution. The 
Table 1. Molar Compositions of Silica Precursor Solutions Used for the Synthesis of a Mesoporous Silica Membrane

$\begin{array}{cc}\text { sample } & \text { TEOS/P123/EtOH/HCl/ } \mathrm{H}_{2} \mathrm{O} \\ 78 \_M & 1: 0.017: 31.8: 0.1: 11.2 \\ 70 \_M & 1: 0.017: 21.2: 0.1: 11.2 \\ 64 \_M & 1: 0.017: 15.9: 0.1: 11.2 \\ 45 \_M & 1: 0.017: 7.43: 0.1: 11.2 \\ \text { 32_M } & 1: 0.017: 4.25: 0.1: 11.2 \\ 19 \_M & 1: 0.017: 2.12: 0.1: 11.2\end{array}$

volumetric composition of ethanol is used for the nomenclature because of the related literature relevant to this work.

Ethanol at 78 vol \% (X = 78) was chosen as the highest concentration in the series on the basis of the original procedure from the literature ${ }^{48}$ For ethanol concentrations of $<19 \mathrm{vol} \%$, the precursor sol gelled and would not flow through the AAO membrane under aspiration. This sets a lower bound $(X=19)$ and is the reason that the addition of ethanol to the precursor solution is required in the synthesis of silica-based membranes via aspiration.

2.3. Characterization by Dynamic Light Scattering (DLS). Dynamic light scattering measurements were made with a NanoBrook Omni particle sizer and a zeta potential analyzer apparatus (Brookhaven Instruments, Long Island, NY, USA) with a $640 \mathrm{~nm}$, $35 \mathrm{~mW}$ red diode laser. Aqueous solutions of Pluronic P123 were prepared and stirred at ambient temperature for $24 \mathrm{~h}$. The P123 solutions were filtered through a $0.22 \mu \mathrm{m}$ Millipore filter and left to equilibrate for up to 3 weeks. Ethanol was added to the solutions as needed. The solutions were centrifuged in order to remove remaining dust particles. Samples of the micellar solutions with ethanol were measured in the DLS apparatus. The intensity correlation function $C(\tau)$ was determined at $90^{\circ}$. The measurements were carried out at 20 and $60{ }^{\circ} \mathrm{C}$.

$C(\tau)$ was analyzed by fitting to an exponential function

$$
C(\tau)=A e^{-2 \Gamma \tau}+B
$$

where $A$ and $B$ are constants specific to the instrument and $\Gamma$ is the relaxation or decay rate.

In the case of a bimodal relaxation rate distribution, the field correlation was fitted to a double-exponential function

$$
C(\tau)=A_{\mathrm{s}} e^{-\Gamma_{\mathrm{s}} \tau}+A_{\mathrm{f}} e^{-\Gamma_{\mathrm{f}} \tau}
$$

where $\Gamma_{\mathrm{s}}$ and $\Gamma_{\mathrm{f}}$ are the relaxation rates of the slow and the fast modes, respectively, and $A_{\mathrm{s}}$ and $A_{\mathrm{f}}$ are their corresponding amplitudes.

The correlation functions were analyzed using the CONTIN method, an algorithm based on the inverse Laplace transform for analyzing the autocorrelation function, ${ }^{49,50}$ to obtain distributions of the relaxation rates $(\Gamma)$.

The diffusion coefficient, $D$, is obtained from the relation

$$
\Gamma=q^{2} D
$$

where $q$ is the length of the scattering vector given by

$$
q=\left(\frac{4 \pi n}{\lambda}\right) \sin \left(\frac{\theta}{2}\right)
$$

where $n$ is the refractive index and $\lambda$ is the laser wavelength.

An effective particle size, namely, the hydrodynamic diameter $\left(D_{\mathrm{h}}\right)$, can then be obtained by using the Stokes-Einstein equation (assuming spherical geometry)

$$
D_{\mathrm{h}}=\frac{k_{\mathrm{B}} T}{3 \pi \eta D}
$$

where $k_{\mathrm{B}}$ is Boltzmann's constant, $T$ is the temperature, $\eta$ is the liquid viscosity, and $D$ is the diffusion coefficient of the particles (all in SI units).

2.4. Molecular Transport of Proteins. The synthesized MSAAM membranes were tested for the molecular transport of proteins,
$\mathrm{Mb}$ and BSA. The membranes were mounted on a filtration apparatus connected to a vacuum pump. The feed solution is a mixture that contains $0.25 \mathrm{mg} / \mathrm{mL}$ of each sample molecule in a $100 \mathrm{mM}$ phosphate buffer solution $(\mathrm{pH}$ 7.0). The permeate solution was analyzed by measuring the ultraviolet absorption spectrum of each species present. The absorbance values were converted to concentration and, consequently, moles of the permeate transported by using Beer-Lambert's law, $A=\varepsilon l c$, where $A$ is the absorbance, $\varepsilon$ is the molar extinction coefficient, $l$ is the path length of the light, and $c$ is the concentration of the species. The absorption measurements were carried out using a Cary $60 \mathrm{UV}$-vis spectrophotometer (Agilent Technologies Ltd., Stockport, U.K.).

2.5. Material Characterization. The synthesized composite membrane and the silica mesostructures formed inside the AAM channels were characterized by a JEOL JSM-6480LV high-performance scanning electron microscope (SEM) (JEOL, Tokyo, Japan). The samples for SEM were sputter-coated with gold for $90 \mathrm{~s}$ to reduce charging. To separately visualize the silica, the composite membrane samples were submerged in $10 \mathrm{wt} \%$ phosphoric acid $\left(\mathrm{H}_{3} \mathrm{PO}_{4}\right)$ for 24 $\mathrm{h}$ to completely dissolve the alumina matrix and obtain the embedded mesoporous silica. Transmission electron microscope (TEM) images were collected by means of a JEOL $2100200 \mathrm{kV}$ TEM (Tokyo, Japan) with a Gatan camera (Pennsylvania, USA). Powder X-ray diffraction (XRD) patterns were measured with a Stadi-P (Stoe, Darmstadt, Germany) with a copper anode and using capillary transmission in the range of $0-5^{\circ}$. Additionally, the samples were characterized with small-angle X-ray scattering (SAXS) in transmission mode with a Ganesha 300XL instrument (SAXSLAB, Copenhagen, Denmark). To determine and analyze the pore structure, nitrogen adsorption and desorption isotherms were measured at $77 \mathrm{~K}$ with an Autosorb-iQMP-XR apparatus (Quantachrome, Florida, USA). Pore size distributions were obtained using the NLDFT model, assuming cylindrical pore geometry. The samples for adsorption/desorption measurements were ground into a powder and degassed at $250{ }^{\circ} \mathrm{C}$ for $8 \mathrm{~h}$ with pretreatment at $100{ }^{\circ} \mathrm{C}$ for $2 \mathrm{~h}$.

\section{RESULTS AND DISCUSSION}

3.1. Textural Property. The mesoporosity of the silica structures formed inside the alumina channels was evaluated by nitrogen adsorption and desorption measurements after calcination of the samples (with the alumina matrix). The isotherms of different silica mesophases formed inside the channels of anodic alumina membranes with decreasing ethanol concentration are shown in Figures 1 and 2. The isotherms of the lower-ethanol-concentration membrane samples display type IV mesopore sorption behavior, ${ }^{51}$ showing capillary condensation at a relative pressure $P / P_{0}$ of between 0.5 and

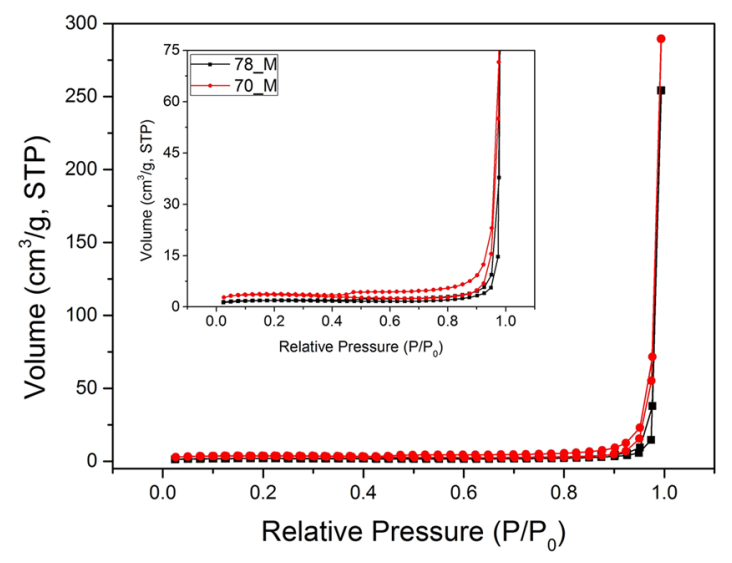

Figure 1. $\mathrm{N}_{2}$ adsorption/desorption isotherms of the different MSAAM membranes formed with their corresponding ethanol volumetric percentages: (-口-) 78_M and (red -0-) 70_M. 


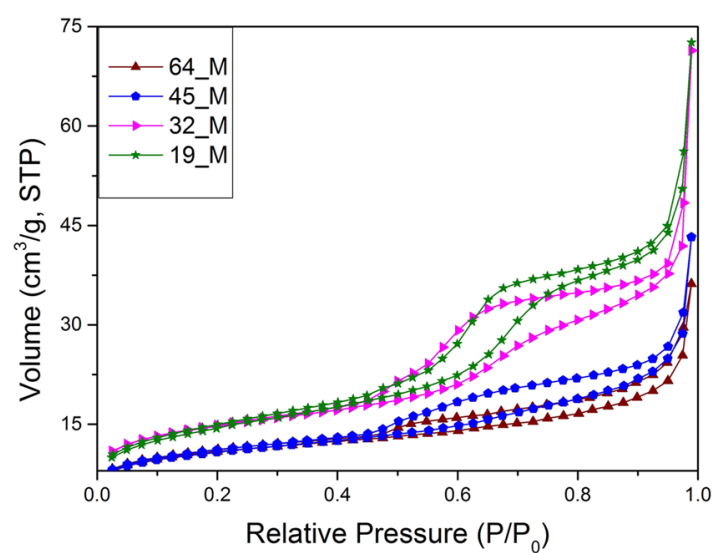

Figure 2. $\mathrm{N}_{2}$ adsorption/desorption isotherms of the different MSAAM membranes formed with their corresponding ethanol volumetric percentages: (brown - $\boldsymbol{\Delta}-$ ) 64_M, (solid blue - - -) 45_M, (pink $-\mathbf{\Delta}-)$ 32_M, and (green $-\star-\overline{-})$ 19_M.

0.7. The relative pressure at which the capillary condensation occurs is shifted to the right to slightly higher values as the ethanol concentration decreases. The isotherms of 78_M and $70 \mathrm{M}$ samples are not of the type IV expected of mesoporous materials because they lack the hysteresis loop and inflection peaks. They can be classified as type II isotherms resulting from the physisorption of nitrogen on macropores.

64_M and 45_M isotherms show hysteresis loops of type $\mathrm{H}_{4}$ according to the IUPAC classification. ${ }^{51}$ The shape of the isotherm indicates the formation of mesoporous silica, possibly with slit-shaped pores, but also the presence of micropores. 32 $\mathrm{M}$ and 19 $\mathrm{M}$ isotherms have well-defined hysteresis loops of types $\mathrm{H}_{2}$ and $\mathrm{H}_{3}$. Their shapes indicate the formation of uniform mesoporous silica. NLDFT analysis shows a narrow pore size distribution (Figure 3) in 19_M obtained from the

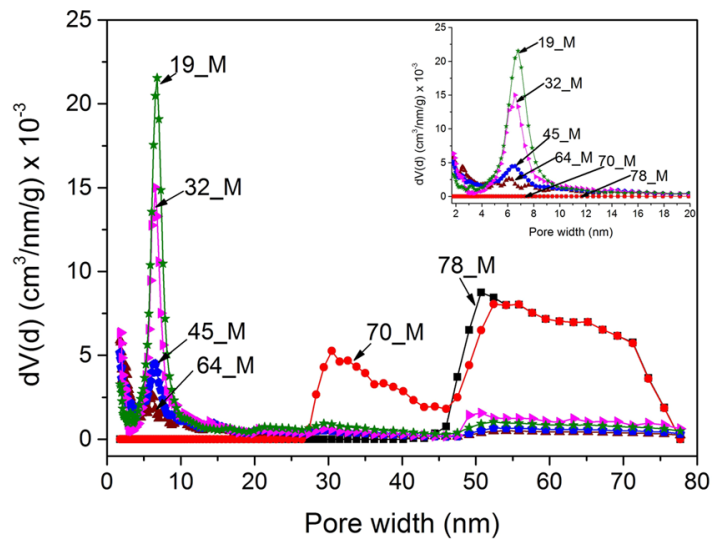

Figure 3. NLDFT pore size distribution of the different MS-AAM membranes formed with their corresponding ethanol volumetric percentages: (-口-) 78_M, (red -0-) 70_M, (brown - $\mathbf{\Delta -}$ ) 64_M, (solid blue - - -) $45 \_\bar{M}$, (pink $-\mathbf{\Delta}-$ ) $32 \_\mathrm{M}$, and (green $-\bar{\star}-$ ) 19_M. Inset: region between 2 and $20 \mathrm{~nm}$.

adsorption branch of the isotherm. The diameter was estimated as $6.8 \mathrm{~nm}$. The very narrow pore size distribution indicates the formation of a uniform and homogeneous silica structure. Results show that increasing the ethanol concentration leads to a broader pore size distribution, with 70_M and 78_M showing the broadest distribution and a few pores in the upper limit of the mesoporous range. The values of the dominant pore diameter (mode) are listed in Table 2. With the narrowing of the pore size distribution, as the ethanol concentration

Table 2. Textural Parameters Evaluated from Nitrogen Adsorption/Desorption Measurements

\begin{tabular}{lccc} 
sample & $\begin{array}{c}\text { BET surface area } \\
\left(\mathrm{m}^{2} / \mathrm{g}\right)\end{array}$ & $\begin{array}{c}\text { pore volume } \\
(\mathrm{cc} / \mathrm{g})\end{array}$ & $\begin{array}{c}\text { pore diameter } \\
(\mathrm{nm})\end{array}$ \\
\hline $78 \_\mathrm{M}$ & 5.5 & 0.39 & 50 \\
$70 \_\mathrm{M}$ & 11 & 0.45 & 30 \\
64_M & 35 & 0.06 & 6.0 \\
$45 \_\mathrm{M}$ & 35 & 0.07 & 6.4 \\
32_M & 48 & 0.11 & 6.6 \\
19_M & 49 & 0.11 & 6.8 \\
\hline
\end{tabular}

decreases, there is a corresponding increase in surface area. The highest value of the BET surface area is obtained for 19_M and is ca. $49 \mathrm{~m}^{2} / \mathrm{g}$. This surface area is per unit mass of the membrane, including both the mesoporous silica and the alumina template. Thus, it is not to be confused with the specific surface area of mesoporous materials themselves, which is much higher (Table $\mathrm{S} 1$ ).

Figure 3 shows that the $78 \mathrm{M}$ and $70 \mathrm{M}$ samples contain much larger pores, even in the range of macropores ( $>50$ $\mathrm{nm}){ }^{51}$ The alumina matrix itself contains macropores of 200 $\mathrm{nm}$ width. These pores are not uniform or consistent in size, as discussed in our earlier work. ${ }^{46}$ Therefore, the high pore diameters (macropores) seen in 78_M and 70_M pore size distributions indicate more empty $\mathrm{AAO}$ channels, whereas for the other samples, the absence of macropores and the narrow mesopore distributions suggest that the AAO pores have been filled with mesoporous silica material.

The samples were also characterized by SAXS (Figure 4). One well-resolved Bragg reflection peak is seen for the

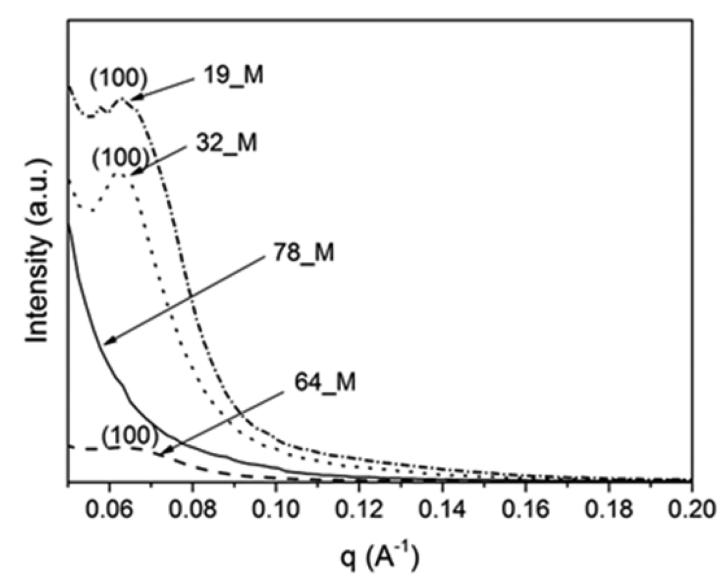

Figure 4. Small-angle X-ray scattering (SAXS) patterns of mesoporous silica-anodic alumina membrane samples synthesized with 78 vol \% (-), 64 vol \% (---), 32 vol \% (...), and 19 vol \% (-.-) ethanol.

membrane samples synthesized with 64,32 , and 19 vol \% ethanol. This peak is indexed as $\left(\begin{array}{lll}1 & 0 & 0\end{array}\right)$. Bragg's law is used to determine the $d$ spacing, $d_{100}$. Figure 4 shows that samples synthesized at the higher ethanol concentration of $78 \mathrm{vol} \%$ do not show a scattering peak. The intensity of the (100) peak increases significantly from the $64 \_M$ pattern to the $32 \_M$ and 19 M patterns. The 64 M peak is also the broadest. The difference in the intensities and the distribution of the peaks indicate an even lower degree of order for the 64_M sample 
and an improvement for the samples with lower ethanol concentration. The appearance of a single peak for all of the patterns may result from insufficient scattering material present (small amount of silica present in the alumina membrane). The absence of higher-order peaks suggests no consistent longrange ordering. All of the samples have approximately the same peak positions (Table 3 ), yielding a very small difference in the

Table 3. Textural Parameters of a Mesoporous SilicaAnodic Alumina Membrane, with Different Ethanol Concentrations, Obtained from SAXS ${ }^{a}$

$\begin{array}{lcccc}\text { sample } & q\left(\mathrm{~nm}^{-1}\right) & d_{100}(\mathrm{~nm}) & a_{0}(\mathrm{~nm}) & \text { pore wall thickness }(\mathrm{nm}) \\ \text { 78_M } & & & & \\ \text { 64_M } & 0.633 & 9.9 & 11.5 & 5.5 \\ \text { 32_M } & 0.614 & 10.2 & 11.8 & 5.2 \\ \text { 19_M } & 0.632 & 9.9 & 11.5 & 4.7\end{array}$

${ }^{a_{\text {The }}}$ cell constant $a_{0}$ was calculated from $d_{100}$, assuming hexagonal order

unit cell constant, $a_{0}$. Because the mesopore diameter, $d_{\mathrm{p}}$, is also very similar, the corresponding silica mesopore wall thickness, $a_{0}-d_{\mathrm{p}}$, is almost the same for ordered samples 19_M, 32_M, and 64_M (Table 3). The unit cell parameter determined for the synthesized membranes with ordered mesoporous silica is consistent with that observed for SBA-15 particles, aged at higher temperatures $\left(>100{ }^{\circ} \mathrm{C}\right) .^{52}$

The Bragg peaks are broad, which may be due to polydispersity and disorder. Crystal defects in a crystalline material could also contribute to peak broadening. Assuming that the broadening arises solely from the crystalline structure, the domain size can be estimated using Scherrer's equation ${ }^{53}$

$$
\text { crystallite size }=\frac{2 \pi K}{\Delta q}
$$

where $K$ is the Scherrer constant ( 0.9 for most materials) and $\Delta q$ is the full width at half-maximum (fwhm) of the peak intensity.

The domain size for $32 \mathrm{M}$ (the most pronounced peak) using the $d_{100}$ peak was then determined to be $7.5 \mathrm{~nm}$. A wellordered silica structure will have a small $\Delta q$ value and thus a larger domain size $(>25 \mathrm{~nm})$. If all of the AAO pores were filled with well-ordered mesoporous silica, three reflection peaks, (100), (110), and (210), associated with hexagonal periodicity would be expected to be seen in the SAXS patterns.

The morphology of the synthesized samples was studied using SEM. Figure 5 shows the SEM images of mesoporous silica-anodic alumina membranes formed with different ethanol concentrations. Evidence of alumina pores filled with silica is seen in the top view. The formation of silica is confirmed after complete etching of the alumina matrix by $10 \%$ $\mathrm{H}_{3} \mathrm{PO}_{4}$ as shown in Figure 6. The silica collected after complete etching of the alumina matrix consists of tubular structures. The SEM images in Figure 6 were analyzed with ImageJ version 1.43 software to determine the dimensions of the tubular structures. The average diameter of the columns ranges from 175 to $278 \mathrm{~nm}$, with longer and thinner structures formed at the lowest ethanol concentration.

These values are of the same order of the anodic alumina pore diameter (around $200 \mathrm{~nm}$ ), as expected. The length of the columns ranges from a few micrometers up to $15 \mu \mathrm{m}$. In addition, the SEM images show that a decrease in the ethanol concentration leads to better-formed and well-defined tubular
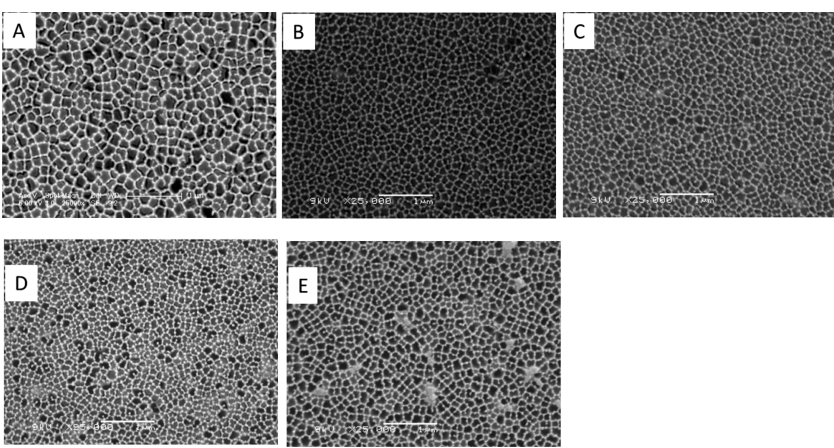

Figure 5. SEM images of MS-AAM membranes formed with a precursor solution of (A) $78 \mathrm{vol} \%$, (B) $64 \mathrm{vol} \%$, (C) $45 \mathrm{vol} \%$, (D) $32 \mathrm{vol} \%$, and (E) 19 vol \% ethanol.
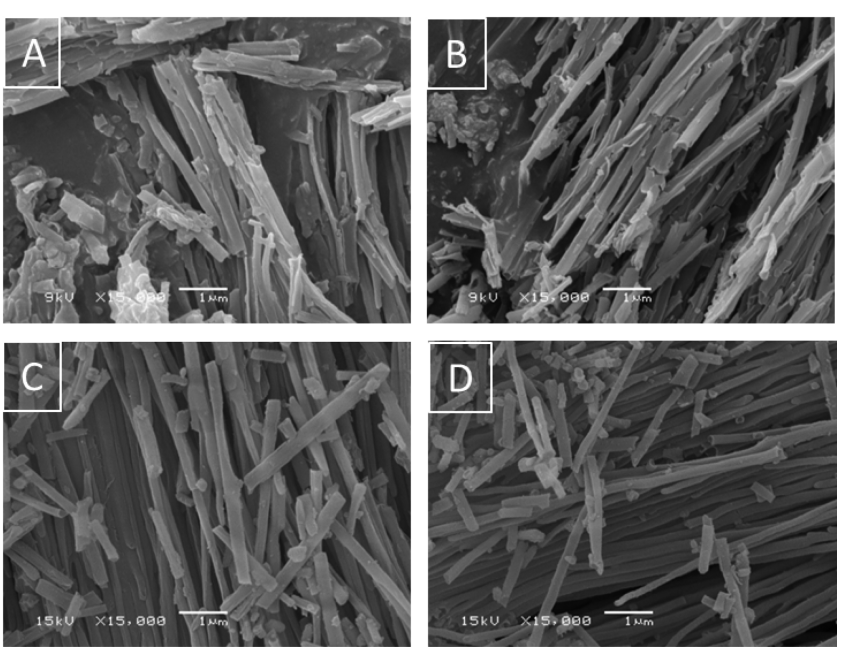

Figure 6. SEM images of silica rods (MS) collected from (A) 78_M, (B) $64 \mathrm{M}$, (C) $32 \mathrm{M}$, and (D) $19 \mathrm{M}$ after complete etching of the alumina matrix with $10 \% \mathrm{H}_{3} \mathrm{PO}_{4}$ for $24 \mathrm{~h}$.

structures. The ordered structure of the collected silica is confirmed by the SAXS patterns shown in Figure 7 for the 19 M and 32_M silicas. The intensity of the 32_M silica Bragg reflection peak is much higher than that for the $19 \mathrm{M}$ silica because of the small quantity of 19 M silica material available for scattering. The peaks for the collected silica occur at the same locations as those for their corresponding composite

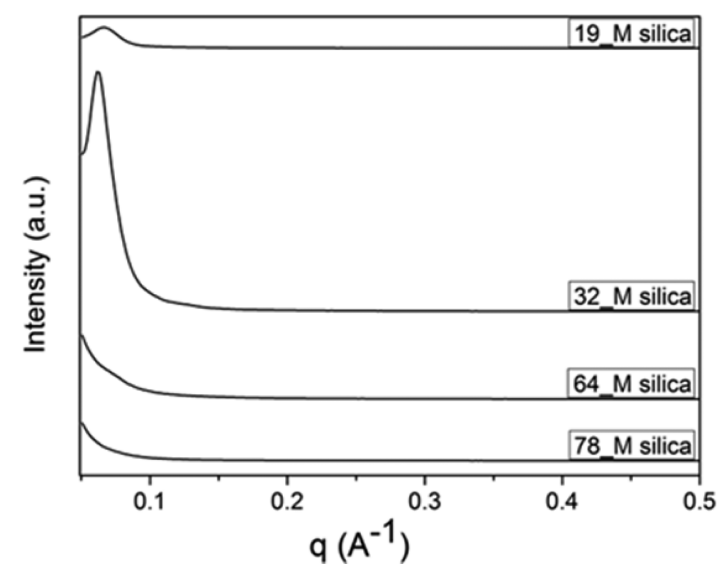

Figure 7. SAXS patterns for silica rods collected after the complete etching of the alumina matrix with $10 \% \mathrm{H}_{3} \mathrm{PO}_{4}$ for $24 \mathrm{~h}$. 
material, i.e., $q=0.61 \mathrm{~nm}^{-1}\left(0.061 \AA^{-1}\right)$ and $q=0.67 \mathrm{~nm}^{-1}$ $\left(0.067 \AA^{-1}\right)$ for $32 \_\mathrm{M}$ silica and $19 \_\mathrm{M}$ silica, respectively.

3.2. Phase Transformations. The local structure, order, and mesophase of the collected silica were further investigated by TEM measurements. The TEM micrographs reveal three types of mesostructures, depending on the ethanol concentration in the precursor solution. For the highest ethanol concentration, $78 \mathrm{vol} \%$, a lamellar arrangement of the pores is observed, where the structure is aligned parallel to the long axis of the columnar silica. Decreasing the ethanol concentration to $64 \mathrm{vol} \%$ leads to a mixture of mesostructures arranged in (mostly) parallel and nonparallel orientations.

Figure $8 \mathrm{~B}$ reveals a phase transition from a lamellar to a hexagonal arrangement of pores. Further decreases in the
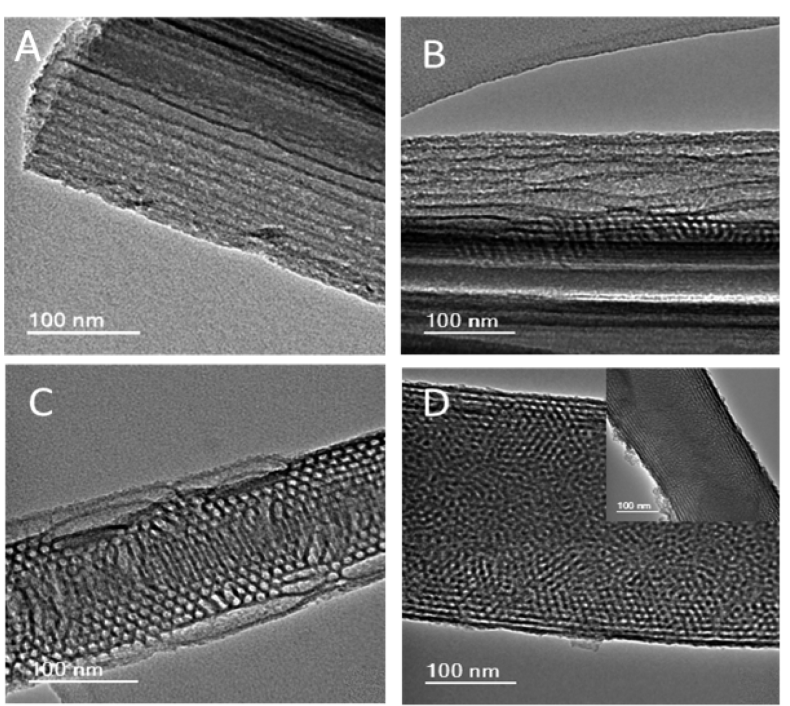

Figure 8. TEM images of silica collected from (A) 78_M, (B) 64_M, (C) 32_M, and (D) 19_M after complete etching of the alumina matrix with $10 \% \mathrm{H}_{3} \mathrm{PO}_{4}$ for $24 \mathrm{~h}$. Inset: columnar pores of 19 M silica.

ethanol content to $32 \mathrm{vol} \%$ leads to a large and homogeneous domain of circular hexagonal pore packing with an estimated pore-to-pore distance of $\sim 10 \mathrm{~nm}$ (estimated from TEM micrographs), in agreement with SAXS results (Table 3). At 19 vol \% ethanol, large domains of both circular and columnar regions exist, as does a combination of circular, columnar hexagonal, and tubular lamellar phases (Figure $8 \mathrm{D}$ and inset). On the basis of the TEM results, the average mesopore sizes of the hexagonally ordered phases of 64_M, 32_M, and 19_M were roughly estimated to be $5.7,6.0$, and $5.9 \overline{\mathrm{nm}}$, respectively. Given the difficulty of estimating the pore size from TEM images, this is in reasonable agreement with the results from the $\mathrm{N}_{2}$ adsorption isotherms (Table 2). It is also observed that the structural order becomes more distorted in the middle region. This is in agreement with the mechanism proposed by Platschek et al. ${ }^{54}$ in which the growth of silica begins at the walls of the alumina channels and moves toward its center. This, therefore, confirms that the alumina walls assist in the growth and orientation of the silica mesopores. Hence, the cosurfactant effect is observed, wherein the higher the alcohol concentration, the lower the surface curvature of the mesostructure obtained. This results in the formation of the lamellar phase at the highest alcohol concentrations, as witnessed earlier.
3.3. DLS Analyses. Denkova et al. proved that ethanol shortens the initiation time $\mathrm{e}^{30}$ of the micelle self-organization and that the addition of ethanol decreases the apparent hydrodynamic radius of triblock copolymers in water. ${ }^{47}$ This explains the need for ethanol in the precursor solutions. The amount of ethanol released by TEOS hydrolysis and the amount of ethanol added are more than sufficient to shorten the self-organization and gelation processes to yield mesoporous silica structures. Our measurements confirm this.

The size (hydrodynamic diameter) of the micelles in the various solutions was calculated from DLS measurements via the Stokes-Einstein equation. The micelles are assumed to have a spherical shape on the basis of their size. ${ }^{37,47,55}$ The hydrodynamic diameter of P123 micelles in pure water was measured to be $18.5 \pm 0.8 \mathrm{~nm}$ and did not change much over a period of 3 weeks (Figure S1).

Figure 9 shows that the addition of $1-15$ vol \% ethanol to a $0.008 \mathrm{~g} / \mathrm{mL}$ P123 micellar solution at $20{ }^{\circ} \mathrm{C}$ causes the

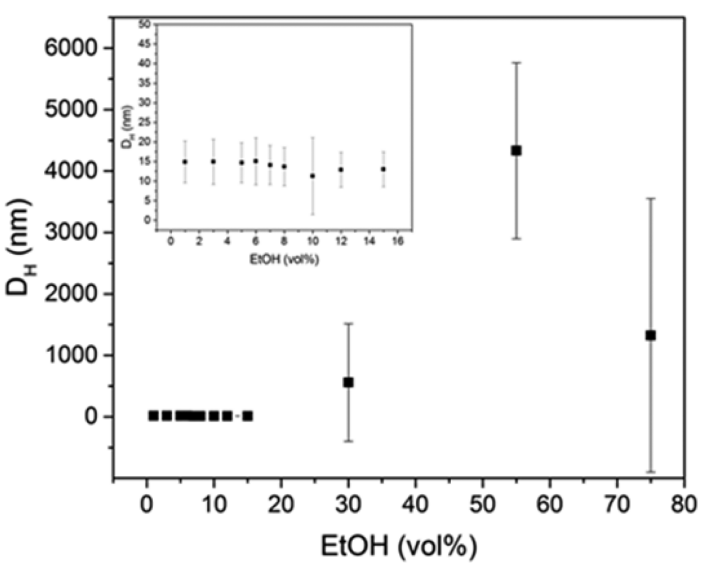

Figure 9. Variation in micelle size, calculated as a hydrodynamic diameter, as a function of ethanol concentration in a $0.008 \mathrm{~g} / \mathrm{mL} \mathrm{P123}$ micellar solution at $20{ }^{\circ} \mathrm{C}$. Inset: region between 0 and 15 vol \% $\mathrm{EtOH}$. Error bars represent the standard deviation of triplicate solutions.

formation of smaller micelles $(15 \pm 6 \mathrm{~nm})$ than those formed by P123 in pure water only. For ethanol concentrations greater than 15 vol \%, the correlation function was fitted using a double-exponential function, eq 2 .

The sharp increase in the calculated hydrodynamic diameter strongly suggests that the assumption of a spherical shape no longer holds true for these micelles. In this case, the fast mode represents the spherical micelles and the slow mode represents growing rodlike micelles. Therefore, at higher ethanol concentrations, the micelles start to grow so that both spherical micelles and elongated micelles exist. The estimated diameter calculations from both the fast and slow modes of the doubleexponential function yielded very large values (on the order of $10^{3} \mathrm{~nm}$ for the slow mode), which are likely a result of larger aggregates. From Figure 10, it can be seen that, at $60{ }^{\circ} \mathrm{C}$, the micelles formed with ethanol become as small as $9.6 \pm 0.7 \mathrm{~nm}$ in diameter. Unlike at $20^{\circ} \mathrm{C}$, the autocorrelation function for the 30 vol \% ethanol micellar solution at $60{ }^{\circ} \mathrm{C}$ can be fitted to a single relaxation fit. The hydrodynamic diameter obtained at the higher temperature is the smallest in the ethanol volume series, $3.5 \pm 0.04 \mathrm{~nm}$. This indicates that the micelles are smaller at the higher temperature of $60{ }^{\circ} \mathrm{C}$. An increase in temperature can induce the dehydration of the ethylene oxide 


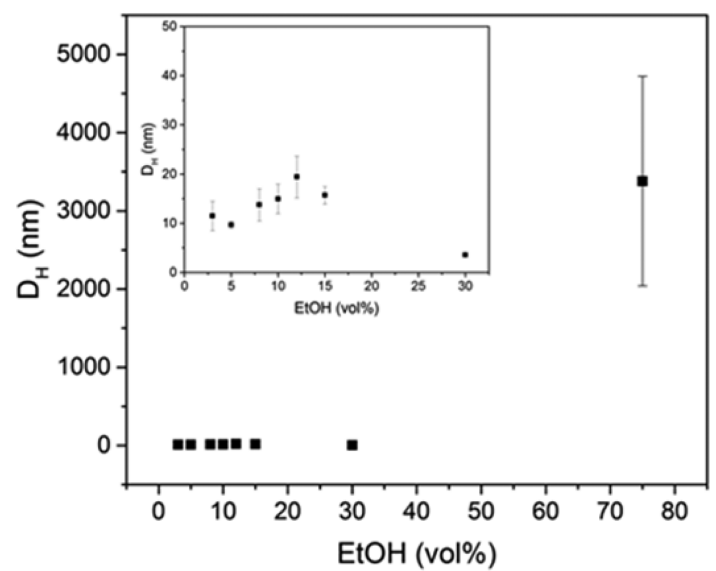

Figure 10. Variation in micelle size, calculated as a hydrodynamic diameter, as a function of different ethanol concentrations in a 0.008 $\mathrm{g} / \mathrm{mL} \mathrm{P} 123$ micellar solution at $60^{\circ} \mathrm{C}$. Inset: the region between 0 and $30 \mathrm{vol} \% \mathrm{EtOH}$. Error bars represent the standard deviation of triplicate solutions.

(EO) chains of the micelle corona. To relate this to the precursor solution for the formation of the composite material, the addition of ethanol decreases the micelle size up to $30 \mathrm{vol}$ $\%$, beyond which the micelles transition from spherical to elongated shapes. These shapes may be wormlike or rodlike, but they both would lead to the formation of larger pores for the silica material in the confined environment.

3.4. Membrane Permeation. The viscosity of the precursor solutions increases as the ethanol concentration decreases from 78_M to $19 \_M$. Consequently, the residence time for the deposition of the sol in the alumina channels increases for the corresponding synthesized membrane samples. This results in a more controlled evaporation-induced selfassembly process for the formation of the silica structures within the confined alumina channels. This correlation (Figure S2) is tested with permeation measurements of the membranes.

The molecular transport of the membranes was tested using protein molecules of different sizes (Figures 11 and 12). In the absence of silica nanostructures, both $\mathrm{Mb}$ and BSA molecules are transported through the AAO membrane (Figure 11A). The presence of the silica nanostructures in 19 M causes a slight decrease in the flux of both molecules. However, $\mathrm{Mb}$

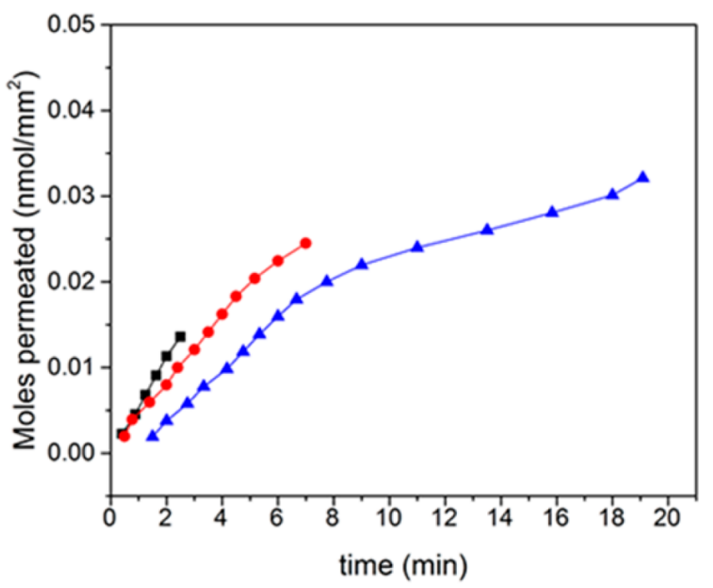

Figure 12. Time-dependent transport of BSA protein molecules through different MS-AAM membranes: (-口-) $70 \mathrm{M}$, (red -0-) 45_M, and (blue $-\mathbf{A}-$ ) 19_M. The standard deviation in the data is within $0.2 \%$ for $70 \_\mathrm{M}, 0.3 \%$ for $45 \_\mathrm{M}$, and $0.5 \%$ for $19 \_\mathrm{M}$.

molecules $(\sim 4.0 \mathrm{~nm})$ and BSA molecules $(\sim 7.2 \mathrm{~nm})$ are transported at nearly the same rate as through the as-purchased alumina membrane (AAO) (Figure 11B). The effective diameter of a BSA molecule is slightly larger than the mean pore size of the silica nanocomposite structures of the 19_M sample, and as such, its transport is expected to be rejected.

However, this is not the case for the transport of BSA through the 19_M membrane. Similarly, BSA transport through $45 \mathrm{M}$ is not rejected. This is because BSA is slightly elongated $\left(5 . \overline{5} \times 5.6 \times 12 \mathrm{~nm}^{3}\right)$, which makes transport through silica pores down to $6 \mathrm{~nm}$ possible. Electrostatic interactions, and not just the size exclusion effect, also play a role. The experiment is carried out at $\mathrm{pH}$ 7. Therefore, $\mathrm{Mb}$ molecules $(\mathrm{pI}=7)$ are neutral, and BSA molecules $(\mathrm{pI}=11)$ are positively charged. Because the silica is negatively charged, attractive interactions may exist between the BSA molecules and silica.

As expected, BSA molecules are transported through 70_M because the mean pore diameter is much larger than that of the BSA molecules. Its flux is lower than that for AAO transport, however, which is indicative of the presence of silica material in the pores of 70_M. The flux of the BSA molecule transport decreases from $\bar{A} A O$ to $70 \_M, 45 \_M$, and finally 19_M. The
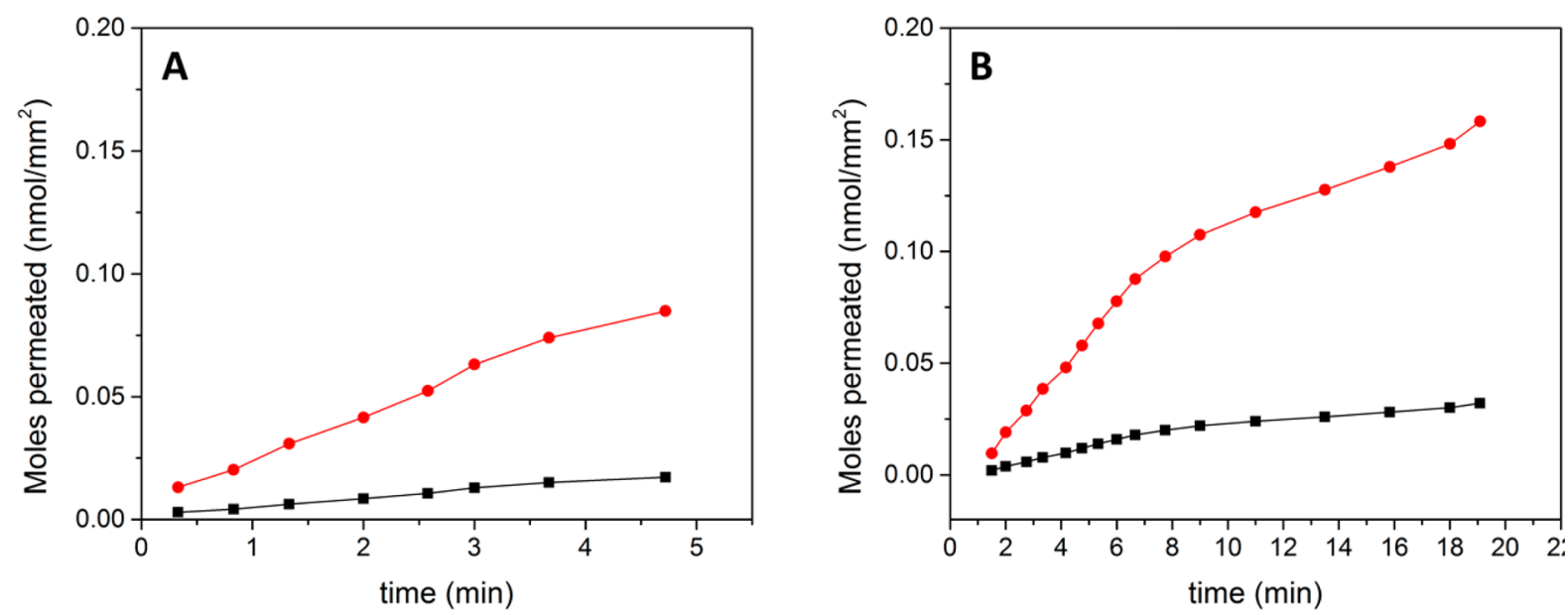

Figure 11. Time-dependent transport of (red - - ) Mb and (- - - ) BSA protein molecules through (A) the AAO membrane and (B) 19_M. The standard deviations are calculated for $(\mathrm{A})$ to be within $0.1 \%$ for $\mathrm{BSA}$ and $0.5 \%$ for $\mathrm{Mb}$ and for (B) to be $0.5 \%$ for $\mathrm{BSA}$ and $2 \%$ for $\mathrm{Mb}$. 
incomplete filling of some alumina pores accounts for the low transport in the 70_M sample. The presence of gaps in the AAO pores is also proposed as a reason for the lack of selectivity in the synthesized membrane samples. It is also seen in Figure 12 that the time taken for the transport of the molecules increases in membrane samples synthesized with decreasing ethanol concentration, indicating that there is a corresponding increased resistance in the membranes. The increased resistance supports the $\mathrm{N}_{2}$ adsorption results that show increasing silica mesoporosity and the amount of silica material with samples synthesized using a lower ethanol concentration. This is also in agreement with Figure 6, which shows the silica nanotubes to be longer and thinner. Unfortunately, the increased mesoporosity is insufficient to permit selective molecular transport in the membranes. This means that the columnar pores coexisting with the circular pores (Figure 8) are not driving transport across the membrane, but their increasing content is responsible for the increased transport time of the molecules.

To further test the functionality of the mesoporous silica in the alumina membrane, $50 \mathrm{~nm}$ gold nanoparticles (Au np) were synthesized according to an established method ${ }^{56}$ and filtered through the membranes. Au np's were chosen because they are rigid and are expected not to change shape or geometry when passing through the membrane pores.

The filtration was carried out under very low vacuum pressure conditions ( $\sim 10 \mathrm{mbar})$. The collected permeate from both samples was measured using UV-vis. The quantitative analysis validates the presence of mesoporous silica in 19_M. The evidence is seen in Figure 13, where the height difference in absorbance indicates blocked Au np's.

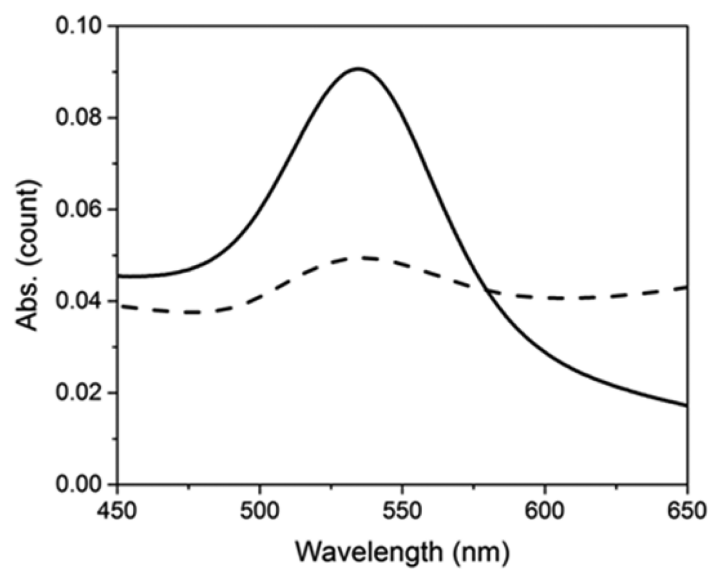

Figure 13. Absorbance spectra of Au np filtered through (-) the aspurchased AAM membrane and (---) 19_M.

\section{CONCLUSIONS}

We have shown that the pore size and morphology of confined silica nanostructures grown in anodic alumina membranes can be controlled by varying the ethanol content in the precursor solution used in the synthesis. The addition of ethanol to the precursor solution influences the final structure of the synthesized membrane and plays an important role in micelle formation and elongation. By decreasing the amount of additional ethanol in the precursor solution, a silica phase that is more ordered with a narrower pore size distribution is formed inside the confined channels of anodic alumina. The amount of ethanol present also affects the rate of formation of the silica-surfactant nanocomposite structures under aspiration by varying the residence time. This study has advanced our understanding of confined mesostructure growth in anodic alumina and the effect of the chemical composition on the growth process to direct orientation and phase order. The proposed protocol can be used to control the synthesis of hierarchically structured inorganic membranes for the separation of enzymes as well as gold nanoparticles from the aqueous solution phase. This is promising and may be useful for applications in separations as well as in catalysis.

\section{ASSOCIATED CONTENT}

\section{S Supporting Information}

The Supporting Information is available free of charge on the ACS Publications website at DOI: 10.1021/acs.langmuir.7b00453.

Explanation and comparison of the specific surface area, DLS data for a micellar solution, and corresponding time taken for the aspiration process to ethanol_MS-AAM (PDF)

\section{AUTHOR INFORMATION}

\section{Corresponding Author}

*E-mail: m.coppens@ucl.ac.uk.

ORCID

Silo Meoto: 0000-0001-9430-9321

\section{Present Address}

${ }^{\dagger}$ Department of Chemical Engineering, University of Utah, Salt Lake City, Utah 84112, United States.

\section{Notes}

The authors declare no competing financial interest.

\section{ACKNOWLEDGMENTS}

Financial support via an EPSRC Frontier Engineering grant to the Centre for Nature Inspired Engineering (EP/K038656/1) is gratefully acknowledged. The authors are also indebted to the Departments of Chemistry and Earth Sciences at UCL for the use of TEM and SEM equipment, respectively.

\section{REFERENCES}

(1) Beck, J. S.; Vartuli, J. C.; Roth, W. J.; Leonowicz, M. E.; Kresge, C. T.; Schmitt, K. D.; Chu, C. T.-W.; Olson, D. H.; Sheppard, E. W.; McCullen, S. B.; et al. A New Family of Mesoporous Molecular Sieves Prepared with Liquid Crystal Templates. J. Am. Chem. Soc. 1992, 114, 10834-10843.

(2) Kresge, C. T.; Leonowicz, M. E.; Roth, W. J.; Vartuli, J. C.; Beck, J. S. Ordered Mesoporous Molecular Sieves Synthesized by a LiquidCrystal Template Mechanism. Nature 1992, 359, 710-712.

(3) Zhao, D.; Feng, J.; Huo, Q.; Melosh, N.; Fredrickson, G. H.; Chmelka, B. F.; D, S. G. Triblock Copolymer Syntheses of Mesoporous Silica with Periodic 50 to 300 Angstrom Pores. Science (Washington, DC, U. S.) 1998, 279, 548-552.

(4) Zhao, D.; Huo, Q.; Feng, J.; Chmelka, B. F.; Stucky, G. D. Nonionic Triblock and Star Diblock Copolymer and Oligomeric Surfactant Syntheses of Highly Ordered, Hydrothermally Stable, Mesoporous Silica Structures. J. Am. Chem. Soc. 1998, 120, 60246036.

(5) Wang, X.; Tseng, Y.-H.; Chan, J. C. C.; Cheng, S. Direct Synthesis of Highly Ordered Large-Pore Functionalized Mesoporous SBA-15 Silica with Methylaminopropyl Groups and Its Catalytic Reactivity in Flavanone Synthesis. Microporous Mesoporous Mater. 2005, 85, 241-251. 
(6) Pitchumani, R.; Li, W.; Coppens, M.-O. Tuning of Nanostructured SBA-15 Silica Using Phosphoric Acid. Catal. Today 2005, $105,618-622$

(7) Azimov, F.; Markova, I.; Stefanova, V.; Sharipov, K. Synthesis and Characterization of SBA-15 and Ti-SBA-15 Nanoporous Materials for DME Catalysts. Univ. Chem. Technol. Metall. 2012, 47, 333-340.

(8) Chang, W.-C.; Deka, J. R.; Wu, H.-Y.; Shieh, F.-K.; Huang, S.-Y.; Kao, H.-M. Synthesis and Characterization of Large Pore Cubic Mesoporous Silicas Functionalized with High Contents of Carboxylic Acid Groups and Their Use as Adsorbents. Appl. Catal., B 2013, 142143, 817-827.

(9) Katiyar, A.; Ji, L.; Smirniotis, P.; Pinto, N. Protein Adsorption on the Mesoporous Molecular Sieve Silicate SBA-15: Effects of $\mathrm{pH}$ and Pore Size. J. Chromatogr. A 2005, 1069, 119-126.

(10) Vivero-Escoto, J. L.; Slowing, I. I.; Trewyn, B. G.; Lin, V. S.-Y. Mesoporous Silica Nanoparticles for Intracellular Controlled Drug Delivery. Small 2010, 6, 1952-1967.

(11) Daiguji, H.; Hwang, J.; Takahashi, A.; Kataoka, S.; Endo, A. Ion Transport in Mesoporous Silica SBA-16 Thin Films with 3D Cubic Structures. Langmuir 2012, 28, 3671-3677.

(12) El-safty, S. A.; Ismail, A. A.; Matsunaga, H.; Nanjo, H.; Mizukami, F. Uniformly Mesocaged Cubic Fd3m Monoliths as Modal Carriers for Optical Chemosensors. J. Phys. Chem. C 2008, 112, 48254835.

(13) El-safty, S. A.; Prabhakaran, D.; Ismail, A. A.; Matsunaga, H.; Mizukami, F. Three-Dimensional Wormhole and Ordered Mesostructures and Their Applicability as Optically Ion-Sensitive Probe Templates. Chem. Mater. 2008, 20, 2644-2654.

(14) Mitchell, D. T.; Lee, S. B.; Trofin, L.; Li, N.; Nevanen, T. K.; Söderlund, H.; Martin, C. R. Smart Nanotubes for Bioseparations and Biocatalysis. J. Am. Chem. Soc. 2002, 124, 11864-11865.

(15) El-Safty, S. A.; Shahat, A.; Warkocki, W.; Ohnuma, M. BuildingBlock-Based Mosaic Cage Silica Nanotubes for Molecular Transport and Separation. Small 2011, 7, 62-65.

(16) Blin, J. L.; Impéror-Clerc, M. Mechanism of Self-Assembly in the Synthesis of Silica Mesoporous Materials: In Situ Studies by X-Ray and Neutron Scattering. Chem. Soc. Rev. 2013, 42, 4071-4082.

(17) Cui, X.; Zin, W.; Cho, W.; Ha, C. Nonionic Triblock Copolymer Synthesis of SBA-15 above the Isoelectric Point of Silica ( $\mathrm{pH}=2-5)$. Mater. Lett. 2005, 59, 2257-2261.

(18) Basumatary, A. K.; Kumar, R. V.; Ghoshal, A. K.; Pugazhenthi, G. Synthesis and Characterization of MCM-41-Ceramic Composite Membrane for the Separation of Chromic Acid from Aqueous Solution. J. Membr. Sci. 2015, 475, 521-532.

(19) Kipkemboi, P.; Fogden, A.; Alfredsson, V.; Flodström, K. Triblock Copolymers as Templates in Mesoporous Silica Formation: Structural Dependence on Polymer Chain Length and Synthesis Temperature. Langmuir 2001, 17, 5398-5402.

(20) Flodström, K.; Alfredsson, V.; Källrot, N. Formation of a New Ia-3d Cubic Meso-Structured Silica via Triblock Copolymer-Assisted Synthesis. J. Am. Chem. Soc. 2003, 125, 4402-4403.

(21) Flodstrom, K. Influence of the Block Length of Triblock Copolymers on the Formation of Mesoporous Silica. Microporous Mesoporous Mater. 2003, 59, 167-176.

(22) Flodström, K.; Wennerström, H.; Alfredsson, V. Mechanism of Mesoporous Silica Formation. A Time-Resolved NMR and TEM Study of Silica-Block Copolymer Aggregation. Langmuir 2004, 20, 680-688.

(23) Zana, R.; Yiv, S.; Strazielle, C.; Lianos, P. Effect of Alcohol on the Properties of Micellar Systems: I. Critical Micellization Concentration, Micelle Molecular Weight and Ionization Degree, and Solubility of Alcohols in Micellar Solutions. J. Colloid Interface Sci. 1981, 80, 208-223.

(24) Hayase, K.; Hayano, S. The Distribution of Higher Alcohols in Aqueous Micellar Solutions. Bull. Chem. Soc. Jpn. 1977, 50, 83-85.

(25) Kim, T.; Kleitz, F.; Paul, B.; Ryoo, R. MCM-48-like Large Mesoporous Silicas with Tailored Pore Structure: Facile Synthesis Domain in a Ternary Triblock Copolymer - Butanol - Water System. J. Am. Chem. Soc. 2005, 127, 7601-7610.
(26) Kleitz, F.; Kim, T.; Ryoo, R. Phase Domain of the Cubic Im3m Mesoporous Silica in the EO106PO70EO106-Butanol-H2O System. Langmuir 2006, 22, 440-445.

(27) Armstrong, J.; Chowdhry, B.; Mitchell, J.; Beezer, A.; Leharne, S. Effect of Cosolvents and Cosolutes upon Aggregation Transitions in Aqueous Solutions of the Poloxamer F87 (Poloxamer P237): A High Sensitivity Differential Scanning Calorimetry Study. J. Phys. Chem. 1996, 100, 1738-1745.

(28) Li, W.; Han, Y.; Zhang, J.; Wang, B. Effect of Ethanol on the Aggregation Properties of Cetyltrimethylammonium Bromide Surfactant *. Colloid J. 2005, 67, 159-163.

(29) Soni, S. S.; Brotons, G.; Bellour, M.; Narayanan, T.; Gibaud, A. Quantitative SAXS Analysis of the P123/Water/Ethanol Ternary Phase Diagram. J. Phys. Chem. B 2006, 110, 15157-15165.

(30) Denkova, A. G.; Mendes, E.; Coppens, M. Kinetics and Mechanism of the Sphere-to-Rod Transition of Triblock Copolymer Micelles in Aqueous Solutions. J. Phys. Chem. B 2009, 113, 989-996.

(31) Sun, J. H.; Moulijn, J. A.; Jansen, J. C.; Maschmeyer, T.; Coppens, M. O. Alcothermal Synthesis under Basic Conditions of an SBA-15 with Long-Range Order and Stability. Adv. Mater. 2001, 13, 327-331.

(32) Landry, C. C.; Tolbert, S. H.; Gallis, K. W.; Monnier, A.; Stucky, G. D.; Norby, P.; O, J. C. H.; York, N. Phase Transformations in Mesostructured Silica/Surfactant Composites. Mechanisms for Change and Applications to Materials Synthesis $\dagger$. Chem. Mater. 2001, 13, 1600-1608.

(33) Liu, S.; Cool, P.; Collart, O.; Voort, P. Van Der; Vansant, E. F.; Lebedev, O. I.; Tendeloo, G. Van; Jiang, M. The Influence of the Alcohol Concentration on the Structural Ordering of Mesoporous Silica: Cosurfactant versus Cosolvent. J. Phys. Chem. B 2003, 107, 10405-10411.

(34) Wang, W.-Q.; Wang, J.-G.; Sun, P.-C.; Ding, D.-T.; Chen, T.-H. Effect of Alcohol on Morphology and Mesostructure Control of Anionic-Surfactant-Templated Mesoporous Silica (AMS). J. Colloid Interface Sci. 2009, 331, 156-162.

(35) Anderson, M. T.; Martin, J. E.; Odinek, J. G.; Newcomer, P. P. Surfactant-Templated Silica Mesophases Formed in Water: Cosolvent Mixtures. Chem. Mater. 1998, 10, 311-321.

(36) Wanka, G.; Hoffman, H.; Ulbricht, W. Phase Diagrams and Aggregation Behaviour of Poly(oxyethylene)-Poly(oxyproprylene)Poly(oxyethylene) Triblock Copolymers in Aqueous Solutions. Macromolecules 1994, 27, 4145-4159.

(37) Nolan, S. L.; Phillips, R. J.; Cotts, P. M.; Dungan, S. R. Light Scattering Study on the Effect of Polymer Composition on the Structural Properties of PEO - PPO - PEO Micelles. J. Colloid Interface Sci. 1997, 191, 291-302.

(38) Klotz, M.; Albouy, P.-A.; Ayral, A.; Ménager, C.; Grosso, D.; Van der Lee, A.; Cabuil, V.; Babonneau, F.; Guizard, C. The True Structure of Hexagonal Mesophase-Templated Silica Films As Revealed by X-Ray Scattering: Effects of Thermal Treatments and of Nanoparticle Seeding. Chem. Mater. 2000, 12, 1721-1728.

(39) Innocenzi, P.; Malfatti, L.; Kidchob, T.; Falcaro, P. OrderDisorder in Self-Assembled Mesostructured Silica Films: A Concepts Review. Chem. Mater. 2009, 21, 2555-2564.

(40) Yamaguchi, A.; Uejo, F.; Yoda, T.; Uchida, T.; Tanamura, Y.; Yamashita, T.; Teramae, N. Self-Assembly of a Silica-Surfactant Nanocomposite in a Porous Alumina Membrane. Nat. Mater. 2004, 3, 337-341.

(41) Platschek, B.; Petkov, N.; Bein, T. Tuning the Structure and Orientation of Hexagonally Ordered Mesoporous Channels in Anodic Alumina Membrane Hosts: A 2D Small-Angle X-Ray Scattering Study. Angew. Chem., Int. Ed. 2006, 45, 1134-1138.

(42) El-Safty, S.; Shahat, A.; Nguyen, H. Nano-Model Membrane Filters for the Well-Controlled Separation of Biomolecules. Colloids Surf., A 2011, 377, 44-53.

(43) Keller, A.; Segal-Peretz, T.; Kauffmann, Y.; Frey, G. L. Control over in-Channel Mesostructure Orientation through AAM Surface Modification. Phys. Chem. Chem. Phys. 2013, 15, 13637-13645. 
(44) Zhang, S.; Wang, Y.; Tan, Y.; Zhu, J.; Liu, K.; Zhu, J.; et al. Anodic Aluminum Oxide with Fine Pore Size Control for Selective and Effective Particulate Matter Filtering. Mater. Res. Express 2016, 3, 074004.

(45) Zhou, L.; Tan, Y.; Wang, J.; Xu, W.; Yuan, Y.; Cai, W.; Zhu, S.; Zhu, J. 3D Self-Assembly of Aluminium Nanoparticles for PlasmonEnhanced Solar Desalination. Nat. Photonics 2016, 10, 393-398.

(46) Meoto, S.; Coppens, M.-O. Anodic Alumina-Templated Synthesis of Mesostructured Silica Membranes - Current Status and Challenges. J. Mater. Chem. A 2014, 2, 5640.

(47) Denkova, A. G.; Mendes, E.; Coppens, M.-O. Effects of Salts and Ethanol on the Population and Morphology of Triblock Copolymer Micelles in Solution. J. Phys. Chem. B 2008, 112, 793-801.

(48) Yamaguchi, A.; Kaneda, H.; Fu, W.; Teramae, N. Structural Control of Surfactant-Templated Mesoporous Silica Formed Inside Columnar Alumina Pores. Adv. Mater. 2008, 20, 1034-1037.

(49) Provencher, S. W. Inverse Problems in Polymer Characterization: Direct Analysis of Polydispersity with Photon Correlation Spectroscopy. Makromol. Chem. 1979, 180, 201-209.

(50) Provencher, S. W. CONTIN_general Purpose Constrained Regularization Program for Inverting Noisy Linear Algebraic and Integral Equations..pdf. Comput. Phys. Commun. 1982, 27, 229-242.

(51) Thommes, M.; Kaneko, K.; Neimark, A. V.; Olivier, J. P.; Rodriguez-Reinoso, F.; Rouquerol, J.; Sing, K. S. W. Physisorption of Gases, with Special Reference to the Evaluation of Surface Area and Pore Size Distribution (IUPAC Technical Report). Pure Appl. Chem. 2015, 87, 1051-1069.

(52) Sang, L.-C.; Vinu, A.; Coppens, M.-O. General Description of the Adsorption of Proteins at Their Iso-Electric Point in Nanoporous Materials. Langmuir 2011, 27, 13828-13837.

(53) Feinle, A.; Lavoie-Cardinal, F.; Akbarzadeh, J.; Peterlik, H.; Adlung, M.; Wickleder, C.; Husing, N. Novel Sol - Gel Precursors for Thin Mesoporous Eu 3+-Doped Silica Coatings as Efficient Luminescent Materials. Chem. Mater. 2012, 24, 3674-3683.

(54) Platschek, B.; Köhn, R.; Döblinger, M.; Bein, T. Formation Mechanism of Mesostructured Silica in Confined Space: An in Situ GISAXS Study. ChemPhysChem 2008, 9, 2059-2067.

(55) Jansson, J.; Schillen, K.; Olofsson, G.; Cardoso, R.; Loh, W. The Interaction between PEO-PPO-PEO Triblock Copolymers and Ionic Surfactants in Aqueous Solution Studied Using Light Scattering and Calorimetry. J. Phys. Chem. B 2004, 108, 82-92.

(56) Perrault, S. D.; Chan, W. C. W. Synthesis and Surface Modification of Highly Monodispersed, Spherical Gold Nanoparticles of 50-200 Nm. J. Am. Chem. Soc. 2009, 131, 17042-17043. 